\title{
Informing: A Cognitive Load Perspective
}

\author{
Slava Kalyuga \\ University of New South Wales, Sydney, NSW, Australia
}

\author{
s.kalyuga@unsw.edu.au
}

\begin{abstract}
In instructional psychology, cognitive load theory has applied knowledge of processing limitations of human cognitive system to enhance effectiveness of instructional procedures and information presentation formats. Underlying assumptions and principles of cognitive load theory are based on a recently proposed evolutionary perspective that regards both human cognitive architecture and biological evolution as natural information processing systems. This paper explores a possibility of extending the established conceptual framework of cognitive load theory to broader processes of informing with the aim of enhancing their effectiveness. The paper discusses general implications of fundamental characteristics of human cognitive architecture for informing science and offers recommendation for improving the process of informing based on cognitive load considerations. Evidence-based guidelines for managing cognitive load when designing and using educational technology tools are also reviewed.
\end{abstract}

Keywords: informing, cognitive load theory, human cognitive architecture, evolutionary perspective, natural information processing systems, instructional psychology, instructional design, sources of cognitive load, ICT-based learning

\section{Introduction}

Informing science investigates how to provide clientele with information in a form, format, and schedule that maximizes its effectiveness (Cohen, 1999; 2009). According to its general, crossdisciplinary definition, information is a feature of objects of different nature that describes their structural aspects represented by patterns attributed to their organization (e.g., Stonier, 1997). Consequently, informing could be regarded as spreading structural patterns (patterns in form) among objects of different nature (Gackowski, 2009). In case of complex information systems such as living organisms (including humans), the aim of informing has been defined as expanding their control over environment (Gackowski, 2009) or, in evolutionary terms, enhancing their chances of survival.

When informing is considered at the level of human information processing, the structure and characteristics of human cognitive architecture could have significant implications for the informing process. Cognitive aspects of human

Material published as part of this publication, either on-line or in print, is copyrighted by the Informing Science Institute. Permission to make digital or paper copy of part or all of these works for personal or classroom use is granted without fee provided that the copies are not made or distributed for profit or commercial advantage AND that copies 1) bear this notice in full and 2) give the full citation on the first page. It is permissible to abstract these works so long as credit is given. To copy in all other cases or to republish or to post on a server or to redistribute to lists requires specific permission and payment of a fee. Contact HPublisher@InformingScience.orgH to request redistribution permission. information processing may critically affect both the informing (informer) and perceiving (client) ends of the informing process. The intersection of cognitive and informing sciences has been related to the area of cognitive informatics (Cohen, 2009).

Cognitive load theory as a branch of instructional psychology (see Sweller, 2003; Sweller, Ayres, \& Kalyuga, 2011, 
for recent overviews) has applied cognitive science to enhance effectiveness and efficiency of instructional design. Since the major aim of instructional design is providing instructional formats and procedures that maximize learning, this could be regarded as a specific area of application of general principles of informing science with learners as clientele. Therefore, instructional and informing sciences are inherently connected. Theoretical frameworks and recommendations of informing science should be applied to instructional design, and established principles of cognitive load theory could also be potentially generalized to advance certain aspects of informing science.

This paper explores a possibility of extending the established conceptual framework and principles of cognitive load theory to broader and more general situations than those in teaching and learning with the aim of enhancing the effectiveness of informing. The paper begins with an overview of major assumptions and principles of cognitive load theory based on a recently proposed evolutionary perspective, describes a corresponding model of human cognitive architecture, followed first by general implications of this architecture to informing science, and then by specific recommendations for improving processes of informing.

\section{Human Cognitive Architecture}

Cognitive load theory describes educational implications of human cognitive architecture (Sweller, 2003, 2004). In its basic assumptions, the theory uses the information processing aspects of biological evolution by natural selection as analogical to basic characteristics of human cognition (Sweller \& Sweller, 2006). It considers both biological evolution and human cognition as examples of a broader category of natural information processing systems. It is assumed that such systems function based on the following five fundamental principles (Sweller, 2003; for an overview, see Sweller et al., 2011):

- $\quad$ The information store principle: natural information processing systems include large stores of information that govern their activities. In human cognitive architecture, longterm memory provides this function.

- $\quad$ The borrowing and reorganizing principle: information in the store is mostly borrowed from other information stores; however, it is reorganized in the process rather than copied exactly. For example, humans imitate other people, listen, and read in order to build long-term memory.

- $\quad$ The randomness as genesis principle: all truly novel (not borrowed) information is acquired by a random generate-and-test process. In the absence of relevant information from other sources, we acquire new information during problem solving by using general methods such as means-ends analysis.

- $\quad$ The narrow limits of change principle: there is a mechanism that prevents large and rapid random changes to the information store that could destroy its functionality. Human cognitive architecture includes a working memory as a major information processor that is severely limited in capacity and duration when dealing with novel information, thus reducing the risk of damaging long-term memory. We can consciously process no more than a few items at a time for no longer than a few seconds. If these limits are exceeded, working memory becomes overloaded and learning inhibited.

- $\quad$ The environmental organizing and linking principle: the information from the store guides behavior of the system in its external environment. When information is used in this role, the above narrow limits of change are effectively reduced or removed. In hu- 
man activities, there are no capacity or duration limits when organized information from long-term memory is transferred to working memory to be used for guiding environmentally appropriate activity.

The above principles were formulated for complex systems such as human cognition or biological evolution. However, structurally relatively less complex non-organic objects could also be regarded as systems with stable patterns of organization. For example, even subatomic particles maintain their identity, physical integrity, and behavioral patterns and engage in information processing that could be described in terms of, at least, some of the above principles. In accordance with the information store principle, they preserve ("store") specific organizational patterns (e.g., specific patterns of atomic shells - electron energy levels in atoms). These patterns may have initially emerged during early stages of cosmogenesis as results of random interactions between particles that under specific conditions, according to the laws of physics, formed relatively more stable patterns (the randomness as genesis principle). Achieving a longer durability in time provided obvious natural testing criteria for the random generate-and-test procedure. According to the environmental organizing and linking principle, these information patterns determine interactions of the systems with their environments (e.g., atomic shells in different atoms determine their interactions in forming more complex molecular structures).

When such systems gradually evolved into more complex natural information processing systems, the narrow limits of change principle emerged to assure that this complexity is not destroyed by large random changes. Complex natural systems evolve mostly incrementally with small changes at a time. Also, complex systems develop the mechanisms of borrowing their structural patterns from other systems (the borrowing and reorganizing principle). The complex systems that rely on the whole set of above principles are flexible and adaptive to environmental changes. They could be therefore related to the class of intelligent natural information processing systems.

The general principles of natural information processing systems could possibly be extended beyond the level of biological evolution that provided the original analogy to the human cognitive architecture. Both biological systems and human cognition are intelligent natural information processing systems, however, as indicated above, some of their principles may also apply to lower-level systems (proto-intelligent systems according to Stonier, 1997). This extended framework is highly speculative in terms of explaining the origin of essential features and capabilities of our mind. However, the similarities between information patterns and processes at different levels of information processing systems are fascinating. This extension may add some new dimension to explaining our ability of reflecting on and uncovering the laws of nature which could be less surprising if our mind is structured in the same way as information processing systems in the rest of the nature.

\section{Cognitive Load Theory}

As an instructional theory, cognitive load theory describes instructional implications of characteristics of human cognitive architecture. The major components of this architecture are long-term memory (a store of information patterns in the form of organized knowledge structures called schemas) and working memory (a conscious information processor and a mechanism for limiting the scope of random changes to the store). In accordance with evolutionary educational psychology (Geary, 2007), cognitive load theory also distinguishes between biologically primary and biologically secondary knowledge. Biologically primary knowledge has been essential for survival of our species throughout the long history of evolution. Therefore, we have evolved to acquire primary knowledge in a rapid, effortless, and mostly automatic way without consciously processing it in working memory. For example, we are predisposed or "hardwired" to acquire 
skills in speaking and listening to our native language, engaging in common social interactions, or applying general problem solving strategies.

Biologically secondary knowledge is associated with relatively recent cultural and technological advancements such as writing and reading skills, science, and technology. Organized forms of education have been established in response to the need of teaching and learning this type of knowledge. Acquisition of secondary knowledge requires effortful and conscious processing in working memory. Therefore, cognitive load theory is concerned primarily with acquisition of biologically secondary knowledge. It should be noted that with sufficient practice, biologically secondary knowledge could also be processed automatically without conscious control and required working memory resources (Schneider \& Shiffrin, 1977). Acquisition of extensive schemas with high levels of automation for some of them is the major goal of instruction. Cognitive load theory generated a variety of effective instructional techniques and procedures (see Sweller et al., 2011, for a recent overview).

According to cognitive load theory, an effective and efficient instructional design creates learning conditions under which learner working memory load is kept within its capacity limits. This could be achieved by eliminating or reducing learner cognitive activities that are not essential for learning and generate unnecessary or wasteful load that is called extraneous cognitive load. Extraneous cognitive load is typically caused by inappropriate instructional formats or procedures introducing unnecessary interacting elements of information to be processed. On the other hand, the theory is also aimed at managing essential for learning load (intrinsic cognitive load) that is determined by interacting elements of information that are essential for achieving specific learning goals.

The capacity of working memory is limited to processing only several new elements or chunks of information at a time (Baddeley, 1986; Cowan, 2001; Miller, 1956). A chunk of information for a particular learner and specific task is determined by the task-relevant schemas the learners hold in their long-term memory. With the development of expertise, the size of a person's chunks increases and many interacting elements for a novice become encapsulated into a single element for an expert. Because intrinsic load determined by interactions between essential elements of information is critical for comprehending the material and constructing knowledge, it is important to provide all the necessary resources for accommodating this load without exceeding working memory limitations.

Extraneous cognitive load is caused by cognitive processes that are not necessary for learning. It is imposed by cognitive activities that a learner is involved in because of the way the learning tasks are designed and presented, rather than because this load is essential for achieving instructional goals. The following situations may cause extraneous cognitive load:

1. Split-attention situations: interacting elements of information are separated over distance (presented at different locations) or time (presented at different times, non-concurrently). Mental integration of these sources of information might require intense search-and-match processes and recall of some elements until other elements are attended and processed.

2. Redundancy situations: two or more sources of information can be understood independently without the need for mental integration. When text simply re-describes a diagram that can be fully understood on its own, processing the text and mentally integrating it with the diagram may result in an extraneous cognitive load. A common form of redundancy is presenting the same information in different modalities, for example, presenting textual explanations in both spoken and written form. 
3. Transiency situations: information disappears before being adequately processed by the learner, for example, when processing lengthy continuous segments of spoken text or animations. Learners need to hold such transient information in their working memory in order to integrate it with the forthcoming related information.

4. Advanced learners situations: levels of learner knowledge in a specific area make the detailed information redundant. Such unnecessary information would distract learners from fluently executing already learned procedures and taking the full advantage of their available knowledge (Kalyuga, 2007). For example, providing fully worked out problem solving steps to advanced learners can generate extraneous cognitive load. Since many interacting elements for a novice become encapsulated into a single schema for an expert, the experienced cognitive load always depends on levels of learner expertise in a specific task domain.

5. Inadequate prior knowledge situations: learners do not have adequate knowledge structures in long-term memory to process new information without cognitive overload. In the absence of relevant knowledge, learners have to resort to general problem solving strategies (e.g., meansends analysis) that generate excessive levels of cognitive load that leaves no working memory resources for meaningful learning.

\section{Implications of Cognitive Load Framework for Informing}

Two major implications of the cognitive load framework for informing are associated with two major components of human cognitive architecture. Firstly, information store (knowledge base) characteristics of informer and client essentially frame informing processes (informer/client framings according to Cohen, 2009). As the major role of information stores in natural information processing systems is to guide the behavior of the system within its environment, the information stores of the informer and client direct their behaviors in the informing process. This role extends to all stages of the process from the informer creating the message to the client interpreting the message, integrating it into the information store (i.e., extracting meaning), and subsequently acting based on it. Information stores provide the sources of bias and determine needs for both the informer and client.

Secondly, both informer and client have severe processing limitations as determined by their working memory capacities (fragility of the system according to Cohen, 2009). However, the magnitude of these limitations depends on the novelty of the processed elements of information relative to the available knowledge base. Therefore, the processing limitations are not fixed but vary depending on the content of the information store that is relevant to the processed information. While even a relatively low number of unfamiliar elements of information may easily overload working memory, a great number of familiar elements of information could be encapsulated into larger chunks based on the available knowledge structures (schemas) and processed in working memory with a minimal cognitive load. Thus, the capacity of information processing channels for both informer and client are flexible and vary continuously depending on the available information structures. This feature critically distinguishes human (or more generally, natural) information processing from artificial (non-natural) systems.

The general framework of informing from the described perspective is depicted in Figure 1. From the point of view of the principles that guide functioning of natural information processing systems, the above two components (long-term memory and working memory) are associated with the information store principle and the narrow limits of change principle. The environmental organizing and linking principle is associated with the role of information structures in directing behavior of the informer and client and effectively reducing working memory limitations when such structures are involved in information processing. What the informers communicate accord- 
ing to their needs would be determined by their actual knowledge structures in long-term memory. On the other hand, what the clients perceive in the message would be defined by their information structures in long-term memory. It could be different interpretations from those intended by informers to the degree their knowledge structures differ. The information structures of both informers and clients provide natural sources of bias and misinformation in the process of informing, including a breakdown in the informing process in case of fundamental differences between their information structures.

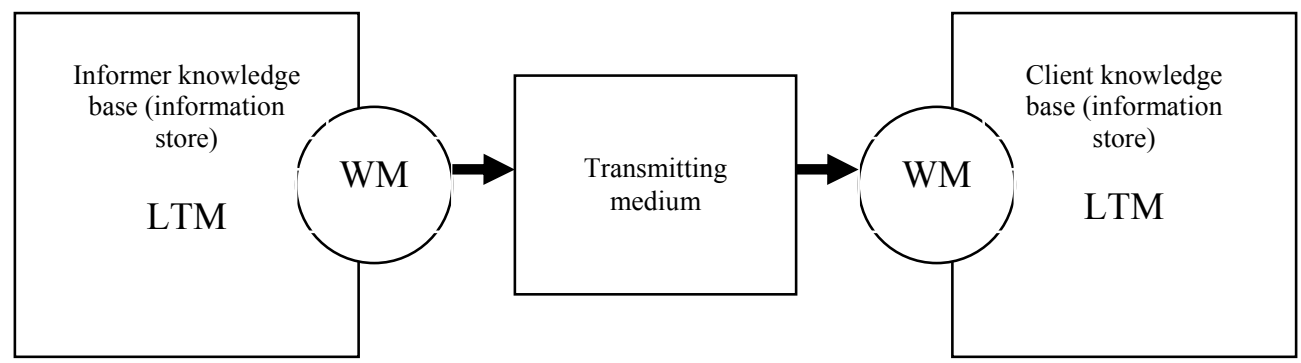

CONTEXT/ENVIRONMENT

Figure 1: General Informing Framework from a Cognitive Load Perspective.

In the absence of relevant knowledge structures to guide the process or parts of it, both the informer and/or the client would engage in search for suitable interpretations based on the random generate-and-test approach to come up with interpretations satisfying their intended goals (the randomness as genesis principle). Therefore, it is essential for an informer to include sufficient level of direct guidance in the informing communication in order to avoid randomly-generated interpretations by clients (especially those lacking relevant information structures) who could misinterpret the intended message. Finally, the borrowing and reorganizing principle is essentially implemented by the process of informing itself, which allows clients to borrow information. At the same time, this information would be inevitably reorganized according to the clients' stored information structures.

What does it mean to "inform a client"? The client could be regarded as "informed" if the communicated information is incorporated (interpreted) within the client's stored information structures. As soon as it is incorporated in the information store, it is ready to be used for guiding behavior in corresponding situations. Information that is incorporated into the information store structures becomes meaningful. It is essentially comprehended by the client, not just registered. The degree of informing could be measured by the degree of changes in the information store structures that could be indirectly measured by the degree of changes in the system's behavior within its environment, both current and possibly modified.

\section{Matching Informing Process with Human Mind}

Based on the above description of potential sources of cognitive load, it is possible to determine cognitive load factors that may influence the process and outcomes of informing. The client's working memory could be overloaded if the combined intrinsic (useful) and extraneous (wasteful) cognitive load exceeds its capacity. In this situation, the first step in dealing with cognitive overload is eliminating or reducing the influence of sources of extraneous cognitive load. The follow- 
ing methods are recommended by cognitive load theory to be used in situations of extraneous cognitive load:

split-attention: physically integrating sources of information that are separated in space or time (e.g., embedding verbal information into diagrams or other textual fragments; synchronizing related sources of information in time), using different modalities (auditory and visual) for presenting verbal and pictorial information;

redundancy: eliminating redundant sources of information (e.g., explaining visual information using spoken only text rather than both spoken and written text simultaneously);

transiency: segmenting lengthy segments of continuous spoken text or animations into smaller portions, or pre-training learners in relevant prior knowledge;

advanced clients: using minimal or reduced levels of non-redundant instructional guidance (e.g., providing problem solving or exploratory environments);

clients with inadequate prior knowledge: using well-guided messages that substitute for missing knowledge structures (e.g., worked-out procedures, explanatory feedback).

If reducing extraneous load still does not eliminate the overload, then additional methods for reducing intrinsic cognitive load should be applied. Among the recommended methods are segmenting the original message into simpler parts, pre-training clients in relevant components of knowledge, and artificially reducing the number of interacting elements of information during the initial phase followed by the message with fully interactive elements of information later.

As noted by Cohen (2009), complexity of information can dramatically impact the nature of informing. Two relevant points could be noted. Firstly, informing in biologically primary knowledge may have very different characteristics from informing in secondary knowledge irrespective of complexity. Secondly, the effectiveness of informing in biologically secondary knowledge critically depends on the relevant content of the information store. From a cognitive load perspective, complexity always depends on levels of client prior knowledge. What is complex (involving high levels of intrinsic cognitive load) for clients with limited relevant content of information store could be simple and routine (low in intrinsic load) for clients with extensive relevant information structures available. The notion of routine performance tasks is directly related to the information store of the performer.

Transience of information is an essential factor in selecting the transmission medium for informing. Just the existence of new technologies cannot be a sufficient reason for their immediate use in informing process. In many situations, due to limitations of human cognitive architecture, transience of auditory text or dynamic visualizations may inhibit informing compared to permanent forms of communication such as written texts or static visuals. In order to effectively use such transmission media, messages may need to be simplified by segmenting or ensuring sufficient levels of client knowledge. If the information deals with biologically primary knowledge, transience may not be a problem, and there could be advantages to speech and animation.

When informing takes place between multiple informers and clients, the cognitive cost of sharing and coordinating information between participants - transaction costs - need to be taken into account (Kirshner, Paas, \& Kirshner, 2009). Group informing potentially has an expanded processing capacity because the intrinsic cognitive load can be effectively subdivided across a number of cooperating working memories. However, transaction costs can offset the advantages of sharing working memory resources, especially for low element interactivity tasks. 


\section{Cognitive Load Perspective and Individual Resonance Model}

In this section, the cognitive load perspective is briefly compared with the individual resonance (or bias filter) model that has been used extensively in informing science to describe the effects of client characteristics on the process of informing (Gill, 2008, 2010; Jamieson \& Hyland, 2006). According to the most recent version of this model (Gill, 2010), before informing messages can be incorporated into client knowledge structures (mental models) held in long-term memory, they pass through several filters that can distort this information by changing, inhibiting, or disproportionately amplifying it. The following five types of filters are suggested: information, cognitive, risk and time preference, motivation, and visceral filters.

The above filters, especially the first three, essentially describe the role of prior knowledge structures (both explicit and implicit knowledge, including various types of available heuristics) in information perception and processing and, consequently, related to managing working memory limitations. From this point of view, cognitive load theory and the resonance model, in effect, use different language for describing the same processes. These two frameworks also use the same underlying human cognitive architecture with interconnected working memory and long-term memory as its major components. Therefore, these two approaches tend to overlap rather than contradict each other.

They may also complement each other in a very fruitful way. For example, the knowledge structures (e.g. heuristics) that introduce different types of biases in informing are well classified and described in the resonance model. This detailed analysis could benefit new research directions within cognitive load theory aimed at developing learner abilities of managing cognitive load in new situations. In many cases, learners may spontaneously abandon effortful (cognitively demanding) processing and search for effective solutions in favor of the effortless use of wellembedded, though inadequate, schemas (e.g., misconceptions in science education). Such mechanisms obviously reduce cognitive load, but at the cost of meaningful learning of relevant knowledge. The analysis of different types of bias-generating heuristics in such situations could be very valuable for this research.

Motivational and affective factors in informing are also developed to a greater degree in the resonance model than the role of such factors in learning and instruction from a cognitive load perspective. Cognitive load theory has mostly focused on factors associated with the cognitive filter in the resonance model. Motivational factors at best have been formally referred to the concept of germane working memory resources ("germane cognitive load") without going into specific instructional means for enhancing such resources. These limitations could be partially explained by the fact that cognitive load theory has been primarily aimed at developing specific instructional techniques for reducing extraneous (unnecessary) load when presenting and formatting instructional messages, with the implicit assumption that learners are well motivated and engaged. In addition, most of the evidence-based techniques and procedures suggested by cognitive load theory have been actually applied primarily to well-defined task domains. The focus of the resonance model on low-structure tasks could be valuable in extending principles of cognitive load theory to ill-defined task areas.

On the other hand, cognitive load theory may also benefit informing science. The theoretical foundations of cognitive load theory, including the evolutionary perspective on human cognitive architecture as a natural information processing system, may provide informing science with a broader outlook, especially in situations when both natural and artificial information processing systems are involved in informing process. Having been aimed at developing specific instructional techniques and procedures (cognitive load effects) for managing cognitive load in instructional situations, cognitive load theory may provide informing science with many established evi- 
dence-based tools for facilitating informing messages that may apply well beyond instructional situations. Some examples of such methods in ICT-based learning are provided in the following section.

\section{Evidence-Based Guidelines for Enhancing ICT-Based Learning}

Cognitive load theory has developed a wide range of instructional principles for reducing extraneous cognitive load and some effective techniques for managing intrinsic cognitive load in general and in ICT-based learning situations (in particular see Kalyuga, 2009; Sweller et al., 2011, for comprehensive overviews). The methods described in the following subsections reduce extraneous cognitive load in learning. In contrast, essential or intrinsic load should either be reduced or increased depending on available cognitive resources and instructional goals. Some techniques recommended for managing intrinsic load are segmenting learning tasks into smaller parts thus reducing intrinsic load by having less information processed at a time, reducing intrinsic load by pre-training learners in essential definitions and procedures prior to the main instructional session, and reducing intrinsic load by learning a limited number of selected isolated elements of information during an initial stage of instruction followed by full-scale instruction involving all interactive elements of information (see Sweller et al., 2011, for a review).

Intrinsic cognitive load could be productively increased by prompting students to self-explain problem-solving steps and procedures using their knowledge of domain principles (selfexplanation effect; e.g., Renkl \& Atkinson, 2003). Another well established method for increasing productive load is to vary the content of learning task by considering different conditions and problem situations rather than similar ones. For example, according to the variability of worked examples effect in cognitive load theory (Paas \& van Merriënboer, 1994), studying examples with different rather than similar surface features results in better and more flexible learning outcomes. In fact, this cognitive load effect was preceded by a contextual interference effect according to which using random sequences of tasks when learning motor skills was more effective than using sequences that are blocked by similarity (Shea \& Morgan, 1979).

In order to maintain concentration and continuously pay attention to presented information, learners should be able to allocate their available working memory resources to processing essential elements of information. In cognitive load theory, such resources devoted to dealing with intrinsic cognitive load are called germane resources. The actual share of working memory resources devoted to productive learning depends not only on the reduction of unnecessary, extraneous cognitive load but, not less importantly, on learners' motivation and engagement. Specific methods and techniques for facilitating learner motivation is a critical factor in learning and instruction that stretches beyond the boundaries of cognitive load theory.

\section{Dealing with Split-Attention and Redundancy}

ICT-based information presentations use different modes and modalities: verbal and pictorial representational modes, and auditory and visual information input modalities. When learners process text and visuals that cannot be understood in isolation, the mental integration of verbal and pictorial representations is required. When text and pictures are not located close to each other or not synchronized in time, integrating these referring representations may increase cognitive load and decrease learning. There are several instructional design techniques for dealing with such split attention situations that may enhance learning. For example, physically integrated or embedded representations were demonstrated to be an effective alternative to "split-source" instructional format (split-attention effect). 
Using dual-modality presentations (e.g., auditory rather than on-screen explanations of a diagram) is an alternative approach to eliminate split attention. Integration of the verbal auditory and pictorial visual information may not overload working memory because its capacity is effectively expanded by using a dual-modality presentation (modality effect). For example, an animation depicting the operation of a technical device with simultaneous audio text may result in better learning than the animation with on-screen text. The modality effect is sufficiently stable and robust. However, simply using audio-visual instructions may not guarantee improved learning if those dual-modality instructions do not reduce extraneous cognitive load. Several conditions required for the modality effect could be identified:

1. Diagrammatic and textual information must refer to each other and be unintelligible, unless they are processed together.

2. Instructional materials should be sufficiently complex with many interacting elements of information.

3. Auditory text should be of limited length (lengthy text is better presented in written form).

4. If diagrams are complex, cuing or signaling (e.g., flashing) may be required so that learners can focus their attention on those parts of the visual display being referred to by the auditory information.

5. Levels of learner expertise should not be high. The effect may be eliminated or reversed with more experienced learners.

Eliminating redundant components of presentations is another method for dealing with potential cognitive overload in ICT-based learning. When redundant onscreen text is embedded into a diagram or narrated at the same time the diagram is presented, it is difficult to avoid processing the redundant information and integrating it with available knowledge structures in long-term memory. These processes consume cognitive resources that become unavailable for constructing higher levels of knowledge. Eliminating redundant verbal or pictorial information could be the best design decision, especially when dealing with more experienced learners. In visual information presentations, an important feature is their fidelity level that characterizes the degree of realism or resemblance to the real world. High fidelity levels with many non-essential details may distract learner attention and impose excessive working memory load. Schematic low-fidelity illustrations could be more effective instructional formats.

The relation between the split-attention and redundancy effects may reverse as the learner gains more expertise. While novice learners may learn best from textual explanations embedded into a diagram or narrated over the diagram, for more experienced learners, diagram-alone materials could generate higher levels of performance and be easier to process. Textual explanations that are essential for novices may become redundant for experts. Thus, the instructional efficiency of different formats of information presentation depends on levels of learner expertise in specific task domains.

\section{Managing Cognitive Load in Interactive Learning Environments}

Sophisticated educational technology tools include various forms of interactivity and respond dynamically to learner actions. They involve multiple representations, linked information networks, and high levels of learner control. High levels of cognitive load in such learning environments could be caused by a large number of variables involved, by uncertainty and non-linear relationships between these variables, and by temporary delays. In many situations, learners have to carry the burden of deciding when to use additional instructional support (if available) and what forms of support to request. While more advanced learners could handle such burden, it may go beyond cognitive resources available to less experienced learners. 
The cognitive load framework could be effectively applied to different forms of dynamic instruction such as animations, simulations, and games. For example, continuous animations may be too cognitively demanding for novice learners due to a high degree of transitivity. Less knowledgeable learners may benefit more from a set of equivalent static diagrams. However, animations still could be relatively more beneficial for more experienced learners who have acquired a sufficient knowledge base for dealing with issues of transitivity and limited working memory capacity (Kalyuga, 2008).

Interactive simulations may provide appropriate environments for exploring various hypotheses and analogies and receiving immediate feedback, thus enhancing the development of problemsolving skills. However, high levels of working memory load could be responsible for instructional failures of many simulations. Many instructional simulations and games represent purely exploratory learning environments with limited guidance for learners. From the cognitive load perspective, random search procedures that novice learners have to use in such environments may impose excessive levels of cognitive load and interfere with meaningful learning. Optimizing levels of instructional guidance represent an essential means for managing cognitive load and enhancing learning outcomes in such environments (Lee, Plass, \& Homer, 2006).

\section{Considering Levels of Learner Expertise}

Most instructional materials are designed in a fixed way with novices as assumed intended learners. However, recent studies of the expertise reversal effect (see Kalyuga, 2007, for a recent overview) have indicated that designs and techniques that are effective with novices can lose their effectiveness and even have negative consequences when used with more experienced learners. When learners do not have sufficient relevant prior knowledge, they have to deal with many new elements of information that may easily overload working memory. These learners may require considerable external support to build new knowledge structures in a relatively efficient manner. On the other hand, more knowledgeable learners may rely on their available domain-specific long-term memory structures for managing cognitive load. Learning procedures and techniques that are beneficial for learners with low levels of prior knowledge may become redundant for more knowledgeable learners. The effect is related to increased cognitive overload for more knowledgeable learners due to processing redundant for these learners instructional components. The major educational technology design implication of these studies is that information presentation and design techniques need to change as learners acquire more expertise in a domain. Well guided instructional presentations that are optimal for novices need to be gradually replaced with less guided instructional formats for more experienced learners.

One method of a gradual transition from fully guided instruction (e.g., worked examples) to problem solving practice is the use of problem completion tasks (van Merriënboer, 1990). A completion task provides a problem statement, a partially worked out solution procedure, with learners required to complete the solution. Completion problems effectively combine a worked-out example with problem solving within one task. Another form of a gradual reduction of instructional guidance as levels of learner expertise increase is the use of faded worked examples (Atkinson, Derry, Renkl, \& Wortham, 2000). With this instructional method, worked-out steps are progressively replaced with problem-solving steps for learners to complete. Faded worked examples thus, in effect, represent a coordinated series of completion problems in which early problems are presented as full worked examples with successive problems requiring learners to complete an increasing number of steps until eventually full problems with no steps already completed is presented.

Tailoring instruction to individual learners is a very complex problem because of many learner characteristics, technical, organizational, and other issues (Kalyuga, 2009). Since prior knowledge is a fundamental cognitive characteristic of learners, the quality of adaptive environments 
depends on the accuracy of information about levels of learner knowledge. Using traditional tests for evaluating such levels may not provide sufficiently precise diagnostic information for detecting different levels of expertise. Also, in order to design adaptive procedures capable of tailoring instruction in real time, it is necessary to have rapid online measures of learner knowledge. The recently suggested idea of rapid diagnostic assessment of expertise is based on evaluating knowledge structures that learners activate rapidly and apply to a briefly presented problem situation (Kalyuga, 2006). Such rapid assessment methods have been successfully used for developing adaptive tutors in several domains (Kalyuga, 2009). The rapid assessment-based approach may offer appropriate tools that combine high levels of diagnostic precision with simplicity of implementation in learner-tailored instruction.

\section{Conclusions}

This paper extended the existing conceptual framework of cognitive load theory that is usually applied to instructional situations to a broader and more general framework of client informing situations. General implications of human cognitive architecture for informing were discussed. Specific recommendations for improving the process of informing include providing sufficient guidance in the absence of relevant knowledge in long-term memory, reducing split-attention, using multiple modalities, eliminating redundancy, taking into account levels of client expertise, considering the evolutionary type of communicated information, transitory nature of information, and cognitive transaction costs in multi-agent informing processes. The paper also described evidence-based guidelines for managing cognitive load when designing and using educational technology tools.

\section{References}

Atkinson, R. K., Derry, S. J., Renkl, A., \& Wortham, D. W. (2000). Learning from examples: Instructional principles from the worked example research. Review of Educational Research, 70, 181-214.

Baddeley, A. D. (1986). Working memory. New York: Oxford University Press.

Cohen, E. (1999). Reconceptualizing information systems as a field of the transdiscipline informing science: From ugly duckling to swan. Journal of Computing and Information Technology, 7(3), 213-219.

Cohen, E. (2009). A philosophy of informing science. Informing Science: The International Journal of an Emerging Transdiscipline, 12, 1-15. Retrieved from http://www.inform.nu/Articles/Vol12/ISJv12p001-015Cohen399.pdf

Cowan, N. (2001). The magical number 4 in short-term memory: A reconsideration of mental storage capacity. Behavioral and Brain Sciences, 24, 87-114.

Gackowski, Z. J. (2009). Informing for operations: Framework, model, and the first principles. Santa Rosa, CA: Informing Science Press.

Geary, D. (2007). Educating the evolved mind: Conceptual foundations for an evolutionary educational psychology. In J. S. Carlson \& J. R. Levin (Eds.), Psychological perspectives on contemporary educational issues (pp. 1-99). Greenwich, CT: Information Age Publishing.

Gill, T. G. (2008). The single client resonance model: Beyond rigor and relevance. Informing Science: The International Journal of an Emerging Transdiscipline, 11, 281-310. Retrieved from http://www.inform.nu/Articles/Vol11/ISJv11p281-310Gill222.pdf

Gill, T. G. (2010). Informing business: Research and education on a rugged landscape. Santa Rosa, CA: Informing Science Press.

Jamieson, K., \& Hyland, P. (2006). Good intuition or fear and uncertainty: The effects of bias on information systems selection decisions. Informing Science: The International Journal of an Emerging Transdiscipline, 9, 49-69. Retrieved from http://www.inform.nu/Articles/Vol9/v9p049-069Jamieson60.pdf 
Kalyuga, S. (2006). Rapid cognitive assessment of learners' knowledge structures. Learning and Instruction, 16, 1-11.

Kalyuga, S. (2007). Expertise reversal effect and its implications for learner-tailored instruction. Educational Psychology Review, 19, 509-539.

Kalyuga, S. (2008). Relative effectiveness of animated and static diagrams: An effect of learner prior knowledge. Computers in Human Behavior, 23, 852-861.

Kalyuga, S. (2009). Managing cognitive load in adaptive multimedia learning. New York: Information Science Reference.

Kirschner, F., Paas, F., \& Kirschner, P. A. (2009). A cognitive load approach to collaborative learning: United brains for complex tasks. Educational Psychology Review, 21, 31-42.

Lee, H., Plass, J. L., \& Homer, B. D. (2006). Optimizing cognitive load for learning from computer-based science simulations. Journal of Educational Psychology, 98, 902-913.

Miller, G. A. (1956). The magical number seven, plus or minus two: Some limits on our capacity for processing information. Psychological Review, 63, 81-97.

Paas, F., \& van Merrienboer, J. J. G. (1994). Variability of worked examples and transfer of geometrical problem-solving skills: A cognitive-load approach. Journal of Educational Psychology, 86, 122-133.

Renkl, A., \& Atkinson, R. K. (2003). Structuring the transition from example study to problem solving in cognitive skills acquisition: A cognitive load perspective. Educational Psychologist, 38, 15-22.

Schneider, W., \& Shiffrin, R. M. (1977). Controlled and automatic human information processing: II. Perceptual learning, automatic attending and a general theory. Psychological Review, 84, 127-190.

Shea, J., \& Morgan, R. L. (1979). Contextual interference effects on the acquisition, retention, and transfer of a motor skill. Journal of Experimental Psychology: Human Learning and Memory, 5, 179-187.

Stonier, T. (1997). Information and meaning: An evolutionary perspective. London: Springer.

Sweller, J. (2003). Evolution of human cognitive architecture. In B. Ross (Ed.), The psychology of learning and motivation (Vol. 43, pp. 215-266). San Diego: Academic Press.

Sweller, J. (2004). Instructional design consequences of an analogy between evolution by natural selection and human cognitive architecture. Instructional Science, 32, 9-31.

Sweller, J., Ayres, P., \& Kalyuga S. (2011). Cognitive load theory. New York: Springer,

Sweller, J., \& Sweller, S. (2006). Natural information processing systems. Evolutionary Psychology, 4, 434-458.

Van Merriënboer, J. J. G. (1990). Strategies for programming instruction in high school: Program completion vs. program generation. Journal of Educational Computing Research, 6, 265-287.

\section{Biography}

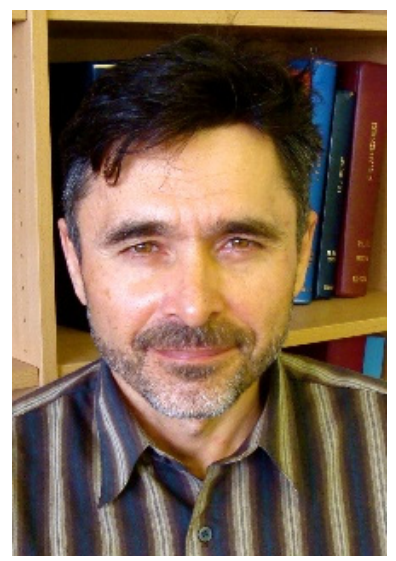

Dr. Kalyuga is Associate Professor at the School of Education, the University of New South Wales, where he received a Ph.D. and has worked since 1995. His research interests are in cognitive processes in learning, cognitive load theory, and evidence-based instructional design principles. His specific contributions include detailed experimental studies of the role of learner prior knowledge in learning (expertise reversal effect), the redundancy effect in multimedia learning, the development of rapid online diagnostic assessment methods, and studies of the effectiveness of different adaptive procedures for tailoring instruction to levels of learner expertise. He is the author of three books and around 50 research articles and chapters. 